\title{
Preoperative prostate health index and \%p2PSA as the significant biomarkers of postoperative pathological outcomes of prostate cancer in Korean males: A prospective multi-institutional study
}

Hongzoo Park ${ }^{1}$ (i), Sang Wook Lee ${ }^{1}$ (i), Geehyun Song ${ }^{1}$ (i), Tae Wook Kang ${ }^{2}$, Jae Hung Jung ${ }^{2}$, Hyun Chul Chung ${ }^{2}$,

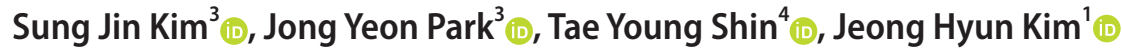

'Department of Urology, Kangwon National University School of Medicine, Chuncheon, ${ }^{2}$ Department of Urology, Wonju Severance Christian Hospital, Yonsei University Wonju College of Medicine, Wonju, ${ }^{3}$ Department of Urology, Gangneung Asan Hospital, University of Ulsan College of Medicine, Gangneung, ${ }^{4}$ Department of Urology,

Hallym University Chuncheon Sacred Heart Hospital, Hallym University College of Medicine, Chuncheon, Korea

Purpose: To evaluate the clinical utility of percentage of serum prostate-specific antigen (proPSA) to free PSA (\%p2PSA) and the prostate health index (PHI) for predicting aggressive pathological outcomes of radical prostatectomy (RP) in Korean males.

Materials and Methods: This prospective observational multicenter study included 160 Korean males who consecutively underwent RP. The predictive utility of preoperative \%p2PSA and PHI for predicting the following pathological outcomes of RP including pT3 disease, pathologic Gleason sum $\geq 7$, and Gleason sum upgrading was investigated using multivariate and decision-curve analyses.

Results: The PHI and \%p2PSA levels were significantly higher in patients with pT3 disease, pathologic Gleason sum $\geq 7$, and Gleason sum upgrading. On univariate analysis, PHI was an accurate predictor of pT3 disease, pathologic Gleason sum $\geq 7$, and Gleason sum upgrading. Multivariate and decision curve analyses revealed that inclusion of $\mathrm{PHI}$ to a base multivariate model including total PSA, percentage free PSA, PSA density, percentage of positive biopsy core, biopsy Gleason sum, and clinical stage factors significantly increased its predictive accuracy; \%p2PSA showed a similar result. However, PHI was a more valuable predictor of pathological outcomes of RP.

Conclusions: This study revealed PHI and \%p2PSA as preoperative biomarkers of pathological outcomes in Korean males who underwent RP for prostate cancer.

Keywords: Pathology; Prostatectomy; Prostatic neoplasms

This is an Open Access article distributed under the terms of the Creative Commons Attribution Non-Commercial License (http://creativecommons.org/licenses/by-nc/4.0) which permits unrestricted non-commercial use, distribution, and reproduction in any medium, provided the original work is properly cited 


\section{INTRODUCTION}

In the prostate-specific antigen (PSA) era, prostate cancer (PCa) screening in males has led to an increase in the identification of PCa patients with low-stage and low-grade disease [1]. However, PSA is unable to distinguish between clinically significant and nonsignificant PCa [2], raising concerns about its overtreatment [3]. Considerable investigations have aimed to overcome the limitations of PSA [4]. Recent studies have shown that [-2]proPSA (p2PSA) and its derivatives, percentage of p2PSA to free PSA (\%p2PSA; [(p2PSA pg/mL)/ (free PSA $n g / m L \times 1,000) \times 100$ ) and the prostate health index (PHI; [p2PSA/free PSA]× $\mathrm{t}$ tPSA) (Beckman Coulter, La Brea, CA, USA) are related to PCa aggressiveness at biopsy [5] and contribute to improving the accuracy of total PSA (tPSA) and percentage of free PSA (\%fPSA) for predicting the presence of $\mathrm{PCa}$ at prostate biopsy [6].

The predictive accuracy of PHI and \%p2PSA with pathologic aggressiveness in PCa patients who underwent radical prostatectomy (RP) has mainly been reported in Western studies [7-10]. Higher PHI and \%p2PSA levels predicted pT3 disease, higher pathologic Gleason sum (GS), and GS upgrading [10,11] A few recent Asian studies involving Chinese males reported on the predictive accuracy of $\mathrm{PHI}$ and \%p2PSA; the results were superior to those of Western studies [12,13].

The incidence of PCa increased in all areas of Asia between 2008 and 2012 [14,15]. However, the incidence and mortality differed significantly across Asia. The PCa mortality rate in the Taiwanese population increased between 1995 and 2006 [16,17], increased in the Korean population between 1983 and 2006 [18], and decreased in Singapore between 1998 and 2006 [19]. The PCa mortality rate in Japan remained stable from 2004 to 2013 [16,20]. The PCa epidemiology in South Korea differed from those of other Asian nations, probably because Korean males have different environmental and genetic factors than other Asian males [16].

In our previous prospective multicenter observational study, we reported that preoperative serum PHI and $\%$ p2PSA levels were more valuable than serum tPSA or $\%$ fPSA levels for predicting the presence of PCa in 246 Korean males who underwent prostate biopsy [21].

Here we evaluated the performance of $\mathrm{PHI}$ and \%p2PSA for predicting final pathological outcomes of RP in Korean males.

\section{MATERIALS AND METHODS}

This prospective observational multicenter study in- cluded four institutions to evaluate the clinical utility of $\mathrm{PHI}$ and \%p2PSA versus the existing biomarkers tPSA and \%fPSA [21].

The study population included 160 consecutive patients who underwent RP for 12-core biopsy-proven PCa between June 2015 and August 2018. Exclusion criteria were a prior history of neoadjuvant androgen-deprivation therapy, use of $5 \alpha$-reductase inhibitors, and conditions that could change the serum p2PSA concentration [21].

Blood samples of the enrolled patients were drawn at least 6 weeks from a prostate biopsy and prior to any manipulations that may cause a transient increase in serum

Table 1. Demographic and clinical characteristics of all study subjects

\begin{tabular}{|c|c|}
\hline Variable & Value $(n=160)$ \\
\hline Age (y) & $68.4 / 69.7(45-79)$ \\
\hline \multicolumn{2}{|l|}{ Clinical stage } \\
\hline $\mathrm{T} 1 \mathrm{c}$ & $117(73.1)$ \\
\hline$\geq \mathrm{T} 2$ & $43(26.9)$ \\
\hline Prostate volume $(\mathrm{mL})$ & $32.2 / 33.3(12-96)$ \\
\hline $\mathrm{tPSA}(\mathrm{ng} / \mathrm{mL})$ & $11.7 / 7.7(3.5-74.9)$ \\
\hline p2PSA & 26.4/18.4 (0.7-189.6) \\
\hline$\% f P S A$ & $0.14 / 0.12(0.01-1.27)$ \\
\hline$\%$ p2PSA & $1.92 / 1.69(0.62-7.3)$ \\
\hline PSA density & $0.28(0.26)$ \\
\hline $\mathrm{PHI}$ & $67.1 / 47.2(19.5-360.9)$ \\
\hline \multicolumn{2}{|l|}{ Biopsy Gleason sum } \\
\hline 6 & $98(61.3)$ \\
\hline $7(3+4)$ & $20(12.5)$ \\
\hline $7(4+3)$ & $33(20.6)$ \\
\hline$\geq 8$ & $9(5.6)$ \\
\hline Number of positive biopsy core & $3.2 / 3(1-12)$ \\
\hline Maximal percentage in each biopsy core (\%) & $45.2 / 39.5(4.6-99.9)$ \\
\hline \multicolumn{2}{|l|}{ Pathology Gleason sum } \\
\hline 6 & $64(40.0)$ \\
\hline $7(3+4)$ & $50(31.3)$ \\
\hline $7(4+3)$ & $31(19.4)$ \\
\hline$\geq 8$ & $15(9.4)$ \\
\hline Gleason upgrading & $34(21.3)$ \\
\hline \multicolumn{2}{|l|}{ Tumor stage } \\
\hline $\mathrm{T} 2$ & $79(49.4)$ \\
\hline T3a & $76(47.5)$ \\
\hline $\mathrm{T} 3 \mathrm{~b}$ & $5(3.1)$ \\
\hline \multicolumn{2}{|l|}{ Resection margin } \\
\hline Negative & $130(81.3)$ \\
\hline Positive & 30 (18.8) \\
\hline
\end{tabular}

Values are presented as mean/median (range), mean (median), or number (\%).

PSA, prostate specific antigen; tPSA, total PSA; fPSA, free PSA; P2PSA, [-2]proPSA; \%fPSA, percentage of fPSA to tPSA; \%p2PSA, percentage of p2PSA to tPSA; PHI, prostate health index. 
biomarker concentrations. The blood samples were processed and managed as described in our previous study [21]. At a single laboratory, the serum samples were analyzed. Experienced genitourinary pathologists evaluated the $\mathrm{RP}$ specimens according to the Stanford protocol [22] and rated $\mathrm{PCa}$ grade according to the 2005 International Society of Urological Pathology consensus [23].

The primary end point of the present study was the clinical utility of preoperative $\mathrm{PHI}$ for predicting postoperative pathological outcomes of PCa. We assessed the presence of pT3, pathologic GS $\geq 7 \mathrm{PCa}$, and GS upgrading. PHI and \%p2PSA were considered the index tests and compared with the established serum biomarkers tPSA, fPSA, and \%fPSA.

Uni- and multivariate logistic regression models were employed to predict the presence of $\mathrm{p}$ T3, pathologic GS $\geq 7$ PCa, and GS upgrading. The factors showing significant results in the univariate analysis were selected as covariates in the multivariate logistic regression models. We plotted the receiver operating characteristic (ROC) and used the area under the ROC curve (AUC) to quantify the predictive accuracy. Each of the variables (\%p2PSA, PHI) was included in the base multivariate model including tPSA, \%fPSA, PSA density, percentage of positive biopsy core, biopsy GS, and clinical stage to assess their ability to determine the three outcomes of interest. We quantified the predictive accuracy gain and used the DeLong method to compare the AUC values [24]. Decision-curve analysis (DCA) was used to evaluate whether the incorporation of \%p2PSA and PHI levels into the base models improved the prognostic model utility [25]. The data of our cohort were analyzed using IBM SPSS Statistics version 25.0 (IBM Corp, Armonk, NY, USA) and R version 3.6.0 for Windows.

The present study protocol was reviewed and approved by the Institutional Review Board of Kangwon National University Hospital (approval number: KNUH 2015-04-004001). Informed consent was obtained from all enrolled subjects.
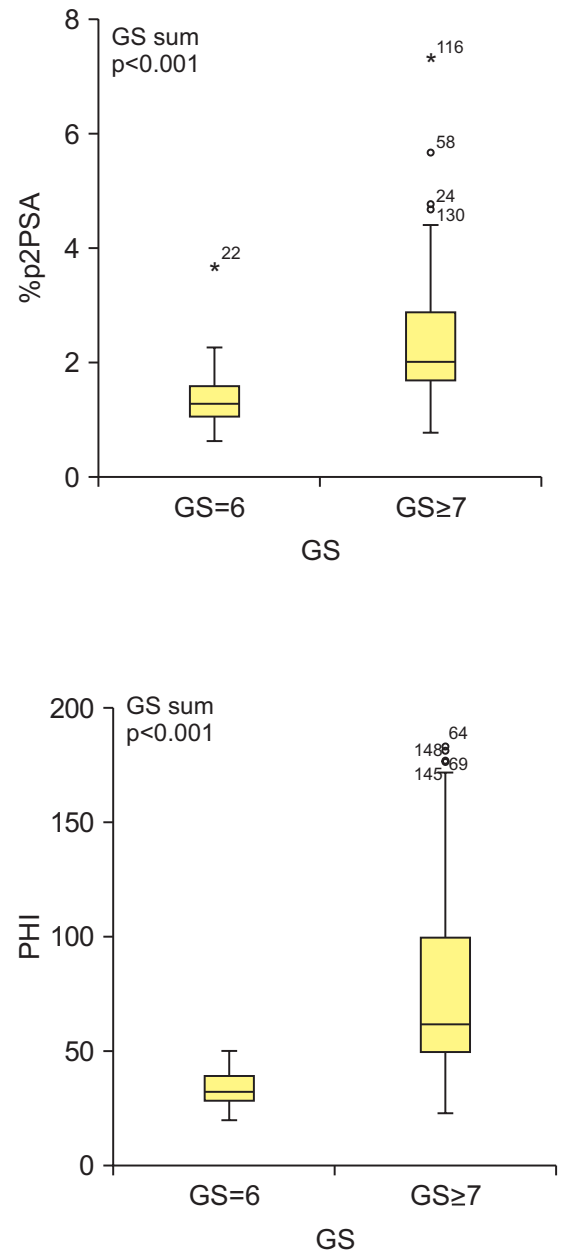
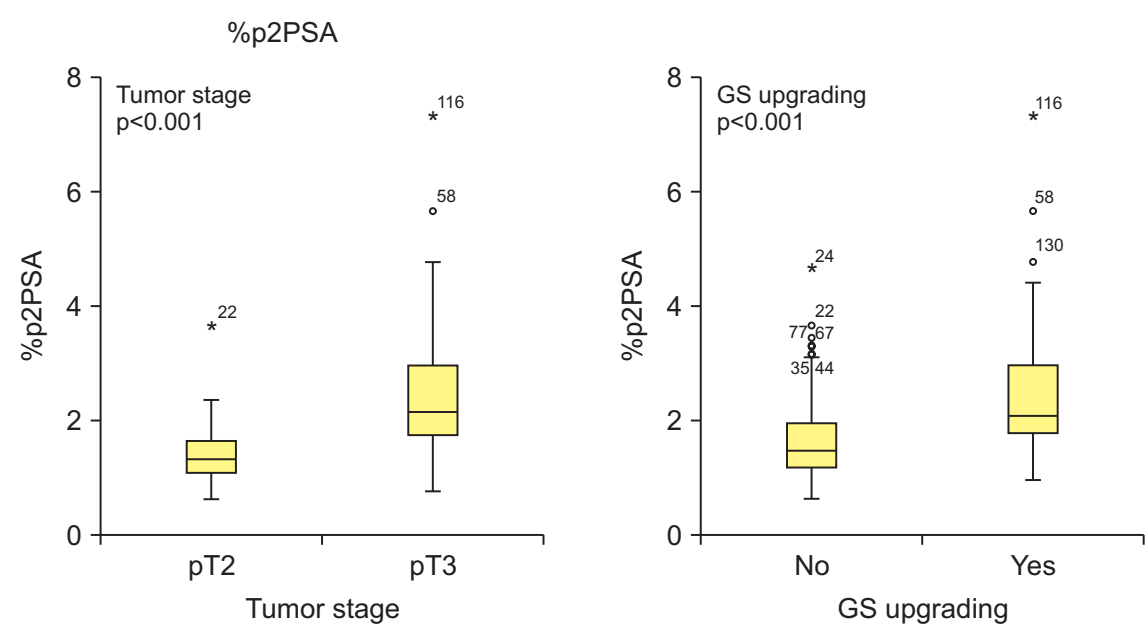

$\mathrm{PHI}$

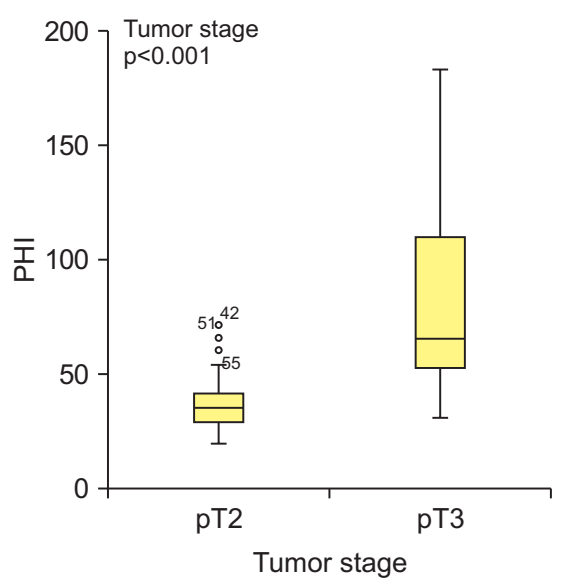

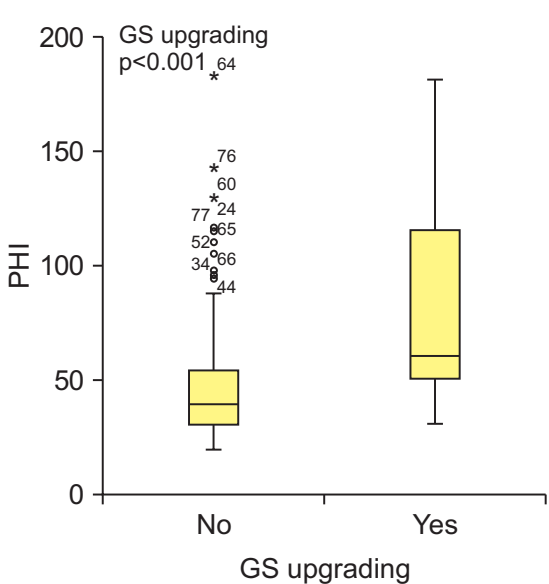

Fig. 1. Percentage of p2PSA to free PSA (\%p2PSA) and prostate health index (PHI) relative to tumor stage, Gleason sum (GS), and GS upgrading. PSA, prostate specific antigen; p2PSA, [-2]proPSA; \%p2PSA, percentage of p2PSA to total PSA. 


\section{RESULTS}

At the four different institutions, 160 patients were enrolled. Table 1 shows the demographic and clinical characteristics of the enrolled patients. The median patient age was 69.7 years (range, 45-79 years). The median PSA level was $7.7 \mathrm{ng} / \mathrm{mL}$ (range, $3.5-74.9 \mathrm{ng} / \mathrm{mL}$ ). Overall, 81 patients (50.6\%) were diagnosed with pT3, 96 (60.0\%) were diagnosed with pathologic GS $\geq 7$, and 34 (21.3\%) showed GS upgrading, which was defined as pathologic GS $\geq 7$ from biopsy GS $=6$.

Fig. 1 shows that patients with pT3 stage, pathologic GS $\geq 7$, and GS upgrading had significantly higher serum \%p2PSA and PHI levels (all $\mathrm{p}<0.001$ ).

Clinical stage (cT1c vs. cT2), percentage of positive biopsy core, biopsy GS, PSA density, tPSA, and \%fPSA were significant predictors of the probability of pT3 at pathologic

Table 2. Univariate and multivariate analysis predicting the probability of pT3 by pathologic stage

\begin{tabular}{|c|c|c|c|c|c|}
\hline \multirow[b]{2}{*}{ Predictor } & \multicolumn{2}{|c|}{ Univariate analysis } & \multicolumn{3}{|c|}{ Multivariate analysis } \\
\hline & $\begin{array}{l}\text { AUC of individual } \\
\text { predictor variables }\end{array}$ & OR; $p$-value & $\begin{array}{c}\text { Base model OR; } \\
\text { p-value }\end{array}$ & $\begin{array}{l}\text { Base model with } \\
\% \text { p2PSA; p-value }\end{array}$ & $\begin{array}{l}\text { Base model with } \\
\text { PHI; } p \text {-value }\end{array}$ \\
\hline Age & 0.428 & $0.977 ; 0.289$ & & & \\
\hline Prostate volume & 0.496 & $1.016 ; 0.093$ & & & \\
\hline Clinical stage & 0.653 & $5.626 ;<0.001$ & $2.726 ; 0.273$ & $2.734 ; 0.381$ & $2.196 ; 0.451$ \\
\hline Percentage of positive biopsy core & 0.598 & $2.105 ;<0.001$ & $1.953 ; 0.083$ & $1.879 ; 0.096$ & $1.882 ; 0.085$ \\
\hline Biopsy Gleason sum & 0.753 & $9.492 ;<0.001$ & $4.493 ; 0.002$ & $1.952 ; 0.059$ & $1.812 ; 0.061$ \\
\hline PSA density & 0.573 & $2.291 ;<0.001$ & $1.156 ; 0.072$ & $1.120 ; 0.125$ & $1.156 ; 0.149$ \\
\hline tPSA & 0.720 & $1.162 ;<0.001$ & $1.157 ; 0.062$ & $1.182 ; 0.045$ & $1.142 ; 0.053$ \\
\hline fPSA & 0.603 & $1.135 ; 0.293$ & & & \\
\hline$\% f P S A$ & 0.321 & $0.001 ;<0.001$ & $0.089 ; 0.568$ & $0.758 ; 0.883$ & $0.074 ; 0.766$ \\
\hline p2PSA & 0.774 & $1.068 ;<0.001$ & & & \\
\hline$\%$ p2PSA & 0.850 & $10.948 ;<0.001$ & & $13.145 ;<0.001$ & \\
\hline PHI & 0.913 & $1.197 ;<0.001$ & & & $1.213 ;<0.001$ \\
\hline AUC of multivariate models & & & 0.849 & 0.939 & 0.951 \\
\hline Gain in predictive accuracy (\%, p-value) & & & & $9.0(<0.001)$ & $10.2(<0.001)$ \\
\hline
\end{tabular}

AUC, area under the curve; OR, odds ratio; PHI, prostate health index; PSA, prostate-specific antigen; tPSA, total PSA; fPSA, free PSA; \% $\mathrm{fPSA}$, the percentage of free PSA to total PSA; \%p2PSA, the percentage of p2PSA to free PSA.

The AUC reflects the predictive values of individual variables (columns) and of the multivariate models in predicting the probability of pT3.

Table 3. Univariate and multivariate analysis predicting the probability of Gleason sum $\geq 7$ at pathologic staging

\begin{tabular}{|c|c|c|c|c|c|}
\hline \multirow[b]{2}{*}{ Predictors } & \multicolumn{2}{|c|}{ Univariate analysis } & \multicolumn{3}{|c|}{ Multivariate analysis } \\
\hline & $\begin{array}{l}\text { AUC of individual } \\
\text { predictor variables }\end{array}$ & OR; p-value & $\begin{array}{l}\text { Base model OR; } \\
\text { p-value }\end{array}$ & $\begin{array}{l}\text { Base model with } \\
\text { \%p2PSA; p-value }\end{array}$ & $\begin{array}{c}\text { Base model with PHI; } \\
\text { p-value }\end{array}$ \\
\hline Age & 0.445 & $0.989 ; 0.623$ & & & \\
\hline Prostate volume & 0.491 & $0.889 ; 0.723$ & & & \\
\hline Clinical stage & 0.698 & $23.109 ;<0.001$ & $43.924 ;<0.001$ & $72.612 ;<0.001$ & $1982.218 ;<0.001$ \\
\hline Percentage of positive biopsy core & 0.637 & $3.251 ; 0.021$ & $7.421 ;<0.001$ & $14.861 ;<0.001$ & $22.176 ;<0.001$ \\
\hline PSA density & 0.582 & $1.023 ; 0.124$ & & & \\
\hline tPSA & 0.714 & $1.180 ;<0.001$ & $1.312 ; 0.038$ & $1.612 ; 0.007$ & $0.991 ; 0.968$ \\
\hline fPSA & 0.570 & $1.060 ; 0.611$ & & & \\
\hline$\%$ fPSA & 0.287 & $0.001 ;<0.001$ & $0.231 ; 0.845$ & $8.239 ; 0.616$ & $0.052 ; 0.790$ \\
\hline p2PSA & 0.754 & $1.063 ;<0.001$ & & & \\
\hline$\%$ p2PSA & 0.836 & $10.557 ;<0.001$ & & & \\
\hline $\mathrm{PHI}$ & 0.849 & $1.243 ;<0.001$ & & & \\
\hline AUC of multivariate models & & & 0.861 & 0.901 & 0.932 \\
\hline Gain in predictive accuracy (\%, p-value) & & & & $4.0 \%(<0.001)$ & $7.1 \%(<0.001)$ \\
\hline
\end{tabular}

AUC, area under the curve; OR, odds ratio; PHI, prostate health index; PSA, prostate-specific antigen; tPSA, total PSA; fPSA, free PSA; \% fPSA, the percentage of free PSA to total PSA; \%p2PSA, the percentage of p2PSA to free PSA.

The AUC reflects the predictive values of individual variables (columns) and of the multivariate models for predicting the probability of Gleason sum $\geq 7$. 
staging on univariate analysis; these factors were selected as covariates in the base model of the multivariate logistic regression analysis (Table 2). Clinical stage, percentage of positive biopsy core, tPSA, and \%fPSA were significant predictors of the probability of pathologic GS $\geq 7$ and GS upgrading on univariate analysis; these factors were selected as covariates in the base models of the multivariate logistic regression analysis (Tables 3,4 ). The uni- and multivariate logistic regression models predicting the probability of pT3 disease (Table 2), pathologic GS $\geq 7$ (Table 3), and GS upgrading (Table 4) revealed \%p2PSA and PHI as independent variables (all $\mathrm{p}<0.001$ ). The inclusion of $\mathrm{PHI}$ or \%p2PSA in the multivariate analyses significantly improved the predictive accuracy of the basic multivariate models. The increase in predictive accuracy was $4.0 \%$ to $13.1 \%$. The ROC curves of these variables are shown in Fig. 2.

Fig. 3 represents the plots of DCA for the models shown in Tables 2-4. Models including PHI clearly resulted in a greater net benefit in pathologic outcome prediction. Net clinical benefit was observed by employing PHI and \%p2PSA to predict pT3, pathologic GS $\geq 7$, or GS upgrading at a threshold probability of $30 \%$ to $70 \%$.

Table 5 shows the sensitivity and specificity at three $\mathrm{PHI}$ levels for predicting pT3, pathologic GS $\geq 7$, and GS up-

Table 4. Univariate and multivariate analyses predicting the probability of Gleason sum upgrading

\begin{tabular}{|c|c|c|c|c|c|}
\hline \multirow[b]{2}{*}{ Predictor } & \multicolumn{2}{|c|}{ Univariate analysis } & \multicolumn{3}{|c|}{ Multivariate analysis } \\
\hline & $\begin{array}{l}\text { AUC of individual } \\
\text { predictor variables }\end{array}$ & OR; p-value & $\begin{array}{c}\text { Base model OR; } \\
\text { p-value }\end{array}$ & $\begin{array}{l}\text { Base model with } \\
\% \text { p2PSA; p-value }\end{array}$ & $\begin{array}{c}\text { Base model with } \mathrm{PHI} ; \\
\text { p-value }\end{array}$ \\
\hline Age & 0.480 & $0.825 ; 0.842$ & & & \\
\hline Prostate volume & 0.510 & $1.005 ; 0.924$ & & & \\
\hline Clinical stage & 0.583 & $2.492 ; 0.031$ & $1.852 ; 0.139$ & $1.153 ; 0.816$ & $1.236 ; 0.773$ \\
\hline Percentage of positive biopsy core & 0.602 & $3.140 ; 0.025$ & $1.125 ; 0.452$ & $1.109 ; 0.529$ & $1.298 ; 0.372$ \\
\hline PSA density & 0.559 & $1.924 ; 0.093$ & & & \\
\hline tPSA & 0.608 & $1.082 ;<0.001$ & $0.976 ; 0.583$ & $0.911 ; 0.480$ & $0.915 ; 0.036$ \\
\hline fPSA & 0.541 & $1.023 ; 0.506$ & & & \\
\hline$\% f P S A$ & 0.392 & $0.001 ; 0.019$ & $0.001 ; 0.046$ & $0.001 ; 0.037$ & $0.001 ; 0.041$ \\
\hline p2PSA & 0.716 & $0.001 ; 1.102$ & & & \\
\hline$\%$ p2PSA & 0.726 & $2.691 ;<0.001$ & & $2.716 ;<0.001$ & \\
\hline $\mathrm{PHI}$ & 0.749 & $1.029 ;<0.001$ & & & $1.032 ;<0.001$ \\
\hline AUC of multivariate models & & & 0.725 & 0.833 & 0.856 \\
\hline Gain in predictive accuracy (\%, p-value) & & & & $10.8 \%(<0.001)$ & $13.1 \%(<0.001)$ \\
\hline
\end{tabular}

AUC, area under the curve; OR, odds ratio; PHI, Prostate Health Index; PSA, prostate-specific antigen; tPSA, total PSA; fPSA, free PSA; \% fPSA, the percentage of free PSA to total PSA; \%p2PSA, the percentage of p2PSA to free PSA.

The AUC reflects the predictive values of individual variables (columns) and of the multivariate models for predicting the probability of Gleason sum upgrading.
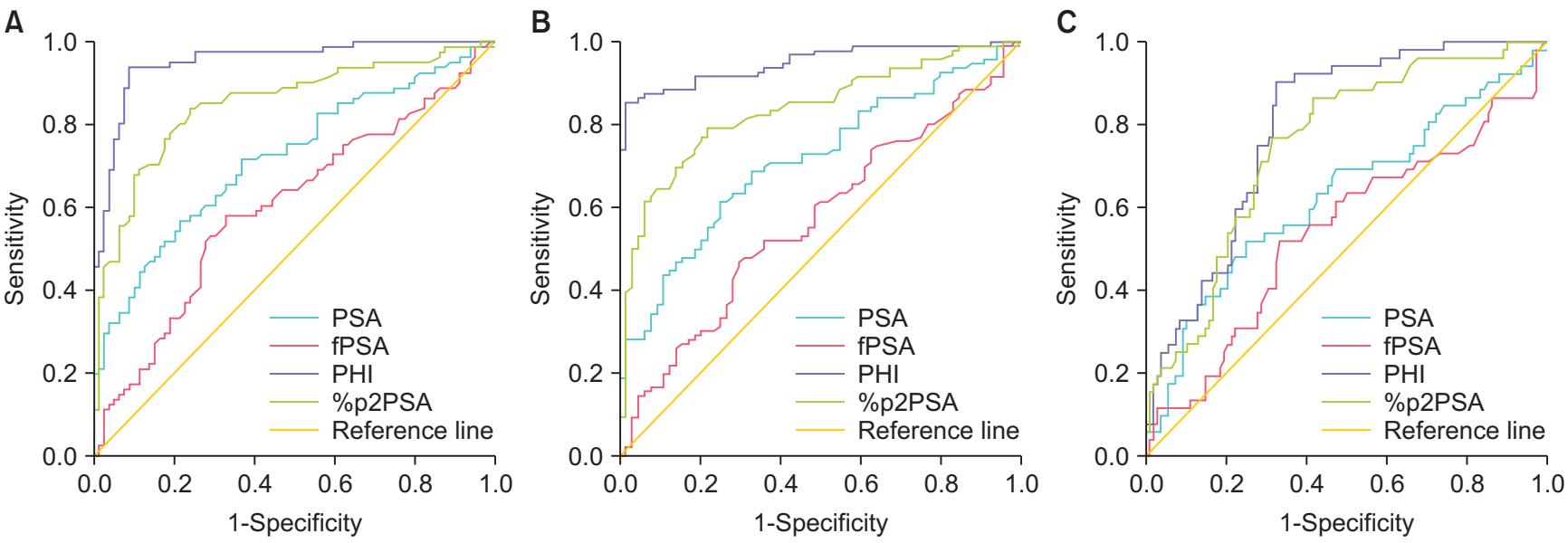

Fig. 2. Receiver operating characteristic curve predicting the probability of $(A) T 3$ at pathologic staging, (B) Gleason sum (GS) $\geq 7$ at pathologic grading, and (C) GS upgrading. PSA, prostate specific antigen; fPSA, free PSA; p2PSA, [-2]proPSA; \%p2PSA, percentage of p2PSA to tPSA; PHI, prostate health index. 

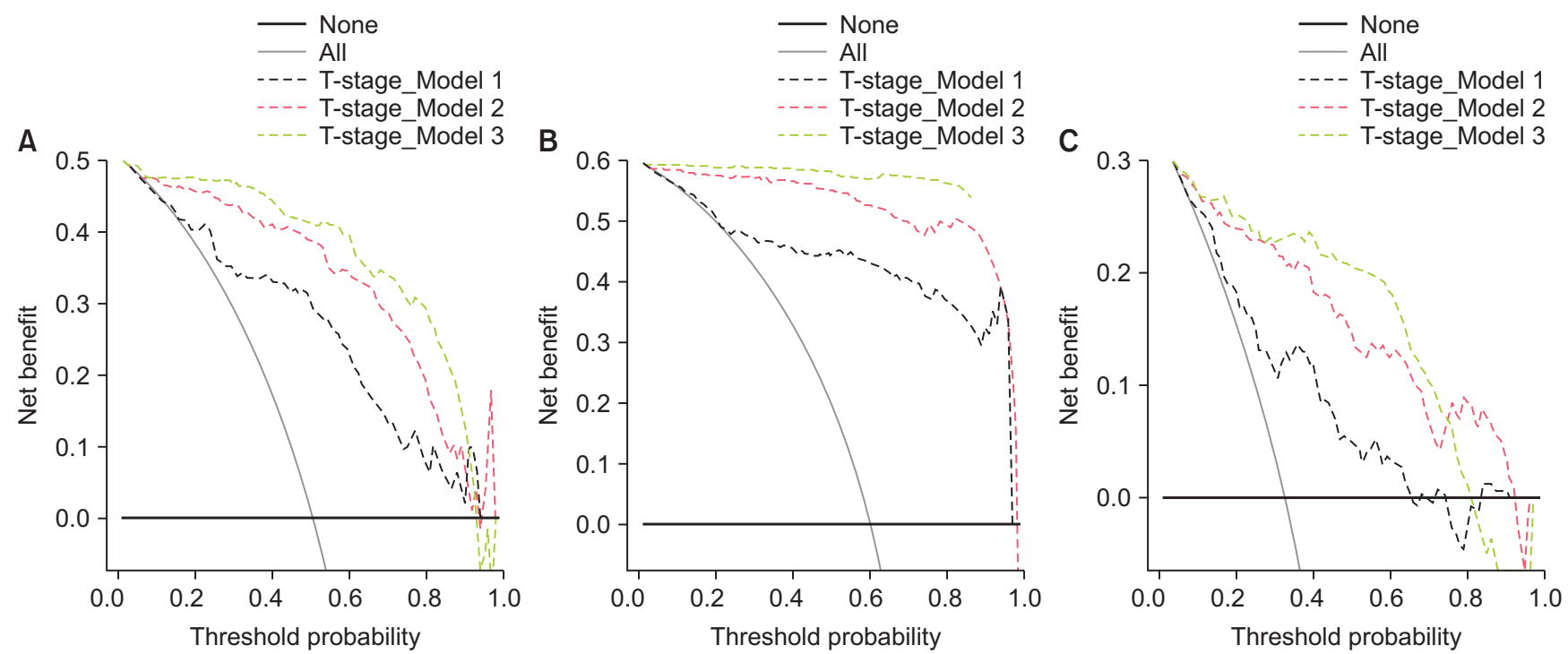

Fig. 3. Decision curve analysis of the effect of the prediction models. The net benefit is plotted against various threshold probabilities. (A) Detection of pT3 at radical prostatectomy. T-stage model 1 is a basic model that includes clinical stage, percentage of positive biopsy core, total prostate-specific antigen (tPSA), free PSA (fPSA), percent fPSA (\%fPSA), biopsy Gleason sum (GS), and PSA density. T-stage model 2 is a basic model that includes all factors of model 1 plus a percentage of p2PSA to fPSA (\%p2PSA). T stage model 3 is a basic model, which includes all factors of model 1 plus the Prostate Health Index (PHI). (B) Detection of pathologic GS $\geq 7$ at radical prostatectomy. GS model 1 is a basic model that includes clinical stage, percentage of positive biopsy core, tPSA, and \%fPSA. GS Model 2 is a basic model that includes all of the factors of model 1 plus \%p2PSA. GS model 3 is a basic model that includes all of the factors of model 1 plus the PHI. (C) Detection of the presence of GS upgrading at radical prostatectomy. Upgrading model 1 is a basic model that includes clinical stage, percentage of positive biopsy core, tPSA, and \%fPSA. Upgrading model 2 is a basic model that includes all of the factors of model 1 plus \%p2PSA. Upgrading model 3 is a basic model that includes all of the factors of model 1 plus the PHI.

Table 5. Sensitivity and specificity at three levels of prostate health index (high sensitivity, best combination, high specificity) for prediction of pT3 disease at pathology, Gleason sum $\geq 7$, and Gleason sum upgrading

\begin{tabular}{lccccc}
\hline \multicolumn{1}{c}{ Prediction } & Criterion & Sensitivity & Specificity & Positive predictive value & Negative predictive value \\
\hline pT3 disease & & & & & \\
& $\geq 30.40$ & $100.0(95.5-100.0)$ & $35.4(25.0-47.0)$ & $61.4(52.5-69.7)$ & $100.0(87.7-100.0)$ \\
& $\geq 45.55$ & $93.8(86.2-98.0)$ & $91.1(82.6-96.4)$ & $91.6(83.4-96.5)$ & $93.5(85.5-97.7)$ \\
& $\geq 71.14$ & $45.7(34.6-57.1)$ & $100.0(95.4-100.0)$ & $100.0(90.5-100.0)$ & $64.2(55.1-72.7)$ \\
Gleason sum $\geq 7$ & & & & & \\
& $\geq 22.29$ & $100.0(96.2-100.0)$ & $7.81(2.6-17.3)$ & $61.9(53.8-69.6)$ & $100.0(47.8-100.0)$ \\
& $\geq 45.55$ & $85.4(76.7-91.8)$ & $98.4(91.6-100.0)$ & $98.8(93.5-100.0)$ & $81.8(71.4-89.7)$ \\
Gleason sum upgrading & $\geq 49.93$ & $73.96(64.0-82.4)$ & $100.0(94.4-100.0)$ & $100.0(94.9-100.0)$ & $71.9(61.4-80.9)$ \\
& & & & & \\
& $\geq 30.19$ & $100.0(94.3-100.0)$ & $23.12(17.2-36.2)$ & $37.2(30.9-49.3)$ & $100.0(86.1-100.0)$ \\
& $\geq 45.92$ & $91.2(72.0-97.5)$ & $66.3(53.2-75.8)$ & $58.1(46.3-69.1)$ & $94.2(84.2-98.1)$ \\
& $\geq 211.23$ & $6.54(2.1-17.2)$ & $100.0(94.1-100.0)$ & $100.0(41.3-100.0)$ & $70.2(62.1-78.2)$ \\
\hline
\end{tabular}

Values are presented as $\%$ (95\% confidence interval).

grading. The best cut-off values of PHI for predicting pT3, pathologic GS, or GS upgrading were 45.55, 45.55, and 45.92, respectively.

\section{DISCUSSION}

The current study shows the benefit of employing PHI and \%p2PSA as preoperative factors for predicting the RP pathological results (pT3, pathologic GS $\geq 7$, or GS upgrading) in cases of localized PCa patients in Korea. The inclusion of PHI and \%p2PSA in multivariate models increased the predictive accuracy from $4.0 \%$ to $13.1 \%$; the models including PHI in DCA exhibited greater net benefit with regard to pathologic outcome probability. DCA is a statistical tool that shows the net benefit of various models known to help predict the outcome in response to the probability of clinical outcome. Clinicians use DCA to determine which model to use to predict clinical outcomes. According to Fig. 3A, for 
example, clinicians in a region with a T3 probability of $20 \%$ to $80 \%$ after prostatectomy can confirm that applying model 2 or 3 can better predict the probability of T3 than model 1 . Our study demonstrated more strong positive results than those of previously reported studies.

Several European reports describe the relationship of PHI, a new biomarker, and RP pathological outcomes. Guazzoni et al. [11] first reported PHI and \%p2PSA as more accurate predictors of $\mathrm{PCa}$ pathological outcomes than previously available markers predicting aggressive $\mathrm{PCa}$ in Italian males. However, the gain in predictive accuracy in their study was lower than our study results, and the models including \%p2PSA and PHI showed no benefit in pT3 prediction in their DCA [11]. Fossati et al. [10] also confirmed \%p2PSA and PHI as significant predictors of pT3 disease and/or pathologic GS $\geq 7$ versus other predictive factors such as PSA, DRE, biopsy, GS, and percentage of positive cores in a multicenter European study of 489 consecutive PCa patients. However, their DCA showed no benefit in pT3 and pathologic GS $\geq 7$ prediction and negative results [10]. Eminaga et al. [9] reported that PHI was not an independent predictor of RP pathological outcomes in German PCa patients in a multivariate analysis.

A few Asian studies have demonstrated the relationship between PHI and pathologic outcome after RP. Chiu et al. [26] reported that both $\mathrm{PHI}$ and \%p2PSA predict aggressive RP pathological outcomes in Chinese males; the study results showed an improved AUC and a net benefit in DCA; however, the gain in predictive accuracy was lower than that of the present study.

To the best of our knowledge, here we demonstrated for the first time in the literature the strong relationship between \%p2PSA, PHI, and aggressive RP pathological outcomes in a Korean cohort. The predictive performance of $\mathrm{PHI}$ and \%p2PSA of our study was superior to that of the Chinese and European cohort [11,26,27]. In the DCA of PHI of our study, a net clinical benefit was seen for pT3, pathologic GS $\geq 7$, and GSn upgrading, and the result was similar to that of a Chinese report [26]. However, the DCA in the two European studies showed no significant net clinical benefit $[10,11]$. The two European cohorts showed much higher proportions (up to 70\%) of patients with clinically aggressive disease (pT3 or pathologic GS $\geq 7$ ) and lower median PSA levels than our cohort $[10,11]$. We postulated that the performance difference of PHI or \%p2PSA between Westerners and Asian Chinese and Koreans might be due to differences in PCa epidemiology (incidence and aggressiveness) and ethnicity. The higher percentage of GS upgrading (32.5\%) compared to other studies may have contributed to the superior
PHI performance in our studies.

Our previous study demonstrated that PHI and \%p2PSA were more valuable biomarkers for predicting the presence of PCa at prostate biopsy than were tPSA and \%fPSA [21]. In the subgroup analysis, these two biomarkers showed superior performance for predicting a GS $\geq 7$ in PCa patients [21]. This result was similar to those of Western and other Asian reports. As a follow-up study, we statistically analyzed the final pathological findings after RP and the relationship with these two biomarkers PHI and \%p2PSA. In particular, we demonstrated the superior clinical utility of $\mathrm{PHI}$ for predicting the probability of GS upgrading, which is one of the biggest concerns when applying active surveillance therapy to the treatment of early PCa. PHI can be a useful clinical biomarker for selecting PCa patients who are subject to active surveillance therapy. We are working on a study to prove this hypothesis.

The strengths of the current study include the following: its prospective observational study, blood processing based on recommended protocol [28], analyses of prostatectomy pathologic findings, and use of DCA [25]. The limitations of the current study include a lack of tumor volume analysis, lack of preoperative MRI finding analysis such as Prostate Imaging Reporting and Data System score, and relatively small sample size. The minimum number of subjects to ensure adequate study power was 150 [29].

\section{CONCLUSIONS}

The current study showed that PHI and \%p2PSA were more powerful than the currently available markers with better predictive accuracy of RP pathological outcomes.

\section{CONFLICTS OF INTEREST}

The authors have nothing to disclose.

\section{ACKNOWLEDGMENTS}

This study has been worked with the support of research grant of Kangwon National University in 2018.

\section{AUTHORS' CONTRIBUTIONS}

Research conception and design: Hongzoo Park and Jeong Hyun Kim. Data acquisition: Hongzoo Park, Sang Wook Lee, Geehyun Song, Tae Wook Kang, Jae Hung Jung, Hyun Chul Chung, Sung Jin Kim, Jong Yeon Park, Tae Young Shin, and Jeong Hyun Kim. Data analysis and inter- 
pretation: Hongzoo Park and Jeong Hyun Kim. Statistical analysis: Hongzoo Park. Drafting of the manuscript: Hongzoo Park and Jeong Hyun Kim. Receiving grant: Hongzoo Park. Approval of final manuscript: all authors.

\section{REFERENCES}

1. Hugosson J, Carlsson S, Aus G, Bergdahl S, Khatami A, Lodding $P$, et al. Mortality results from the Göteborg randomised population-based prostate-cancer screening trial. Lancet Oncol 2010;11:725-32.

2. McGrath S, Christidis D, Perera M, Hong SK, Manning T, Vela I, et al. Prostate cancer biomarkers: are we hitting the mark? Prostate Int 2016;4:130-5.

3. Moyer VA; U.S. Preventive Services Task Force. Screening for prostate cancer: U.S. Preventive Services Task Force recommendation statement. Ann Intern Med 2012;157:120-34.

4. Hori S, Blanchet JS, McLoughlin J. From prostate-specific antigen (PSA) to precursor PSA (proPSA) isoforms: a review of the emerging role of proPSAs in the detection and management of early prostate cancer. BJU Int 2013;112:717-28.

5. Hendriks RJ, van Oort IM, Schalken JA. Blood-based and urinary prostate cancer biomarkers: a review and comparison of novel biomarkers for detection and treatment decisions. Prostate Cancer Prostatic Dis 2017;20:12-9.

6. Sriplakich S, Lojanapiwat B, Chongruksut W, Phuriyaphan S, Kitirattakarn P, Jun-Ou J, et al. Prospective performance of the Prostate Health Index in prostate cancer detection in the first prostate biopsy of men with a total prostatic specific antigen of 4-10 ng/mL and negative digital rectal examination. Prostate Int 2018;6:136-9.

7. Jansen FH, van Schaik RH, Kurstjens J, Horninger W, Klocker $\mathrm{H}$, Bektic J, et al. Prostate-specific antigen (PSA) isoform p2PSA in combination with total PSA and free PSA improves diagnostic accuracy in prostate cancer detection. Eur Urol 2010;57:921-7.

8. Guazzoni G, Nava L, Lazzeri M, Scattoni V, Lughezzani G, Maccagnano C, et al. Prostate-specific antigen (PSA) isoform p2PSA significantly improves the prediction of prostate cancer at initial extended prostate biopsies in patients with total PSA between 2.0 and $10 \mathrm{ng} / \mathrm{ml}$ : results of a prospective study in a clinical setting. Eur Urol 2011;60:214-22.

9. Eminaga O, Bögemann M, Breil B, Titze U, Wötzel F, Eltze E, et al. Preoperative prostate-specific antigen isoform p2PSA $\leq 22.5$ $\mathrm{pg} / \mathrm{ml}$ predicts advanced prostate cancer in patients undergoing radical prostatectomy. Urol Oncol 2014;32:1317-26.

10. Fossati N, Buffi NM, Haese A, Stephan C, Larcher A, McNicholas T, et al. Preoperative prostate-specific antigen isoform p2PSA and its derivatives, \%p2PSA and prostate health index, predict pathologic outcomes in patients undergoing radical prostatectomy for prostate cancer: results from a Multicentric European Prospective Study. Eur Urol 2015;68:132-8.

11. Guazzoni G, Lazzeri M, Nava L, Lughezzani G, Larcher A, Scattoni V, et al. Preoperative prostate-specific antigen isoform p2PSA and its derivatives, \%p2PSA and prostate health index, predict pathologic outcomes in patients undergoing radical prostatectomy for prostate cancer. Eur Urol 2012;61:455-66.

12. Chiu PK, Ng CF, Semjonow A, Zhu Y, Vincendeau S, Houlgatte A, et al. A multicentre evaluation of the role of the Prostate Health Index (PHI) in regions with differing prevalence of prostate cancer: adjustment of PHI reference ranges is needed for European and Asian settings. Eur Urol 2019;75:558-61.

13. Tang B, Han CT, Lu XL, Wan FN, Zhang CZ, Zhu Y, et al. Preoperative prostate health index predicts poor pathologic outcomes of radical prostatectomy in patients with biopsy-detected low-risk patients prostate cancer: results from a Chinese prospective cohort. Prostate Cancer Prostatic Dis 2018;21:6470.

14. Ferlay J, Soerjomataram I, Dikshit R, Eser S, Mathers C, Rebelo $\mathrm{M}$, et al. Cancer incidence and mortality worldwide: sources, methods and major patterns in GLOBOCAN 2012. Int J Cancer 2015;136:E359-86.

15. Ferlay J, Shin HR, Bray F, Forman D, Mathers C, Parkin DM. Estimates of worldwide burden of cancer in 2008: GLOBOCAN 2008. Int J Cancer 2010;127:2893-917.

16. Ha Chung B, Horie S, Chiong E. The incidence, mortality, and risk factors of prostate cancer in Asian men. Prostate Int 2019;7:1-8.

17. Tseng CH. Prostate cancer mortality in Taiwanese men: increasing age-standardized trend in general population and increased risk in diabetic men. Ann Med 2011;43:142-50.

18. Park SK, Sakoda LC, Kang D, Chokkalingam AP, Lee E, Shin $\mathrm{HR}$, et al. Rising prostate cancer rates in South Korea. Prostate 2006;66:1285-91.

19. Chen C, Naidoo N, Yang Q, Hartman M, Verkooijen HM, Loy EY, et al. A comparative population-based study of prostate cancer incidence and mortality rates in Singapore, Sweden and Geneva, Switzerland from 1973 to 2006. BMC Cancer 2012;12:222.

20. Katanoda K, Hori M, Matsuda T, Shibata A, Nishino Y, Hattori $\mathrm{M}$, et al. An updated report on the trends in cancer incidence and mortality in Japan, 1958-2013. Jpn J Clin Oncol 2015;45:390-401.

21. Park H, Lee SW, Song G, Kang TW, Jung JH, Chung HC, et al. Diagnostic performance of \%[-2]proPSA and Prostate Health Index for prostate cancer: prospective, multi-institutional study. J Korean Med Sci 2018;33:e94.

22. van der Kwast TH, Amin MB, Billis A, Epstein JI, Griffiths 
D, Humphrey PA, et al.; ISUP Prostate Cancer Group. International Society of Urological Pathology (ISUP) consensus conference on handling and staging of radical prostatectomy specimens. Working group 2: T2 substaging and prostate cancer volume. Mod Pathol 2011;24:16-25.

23. Epstein JI, Allsbrook WC Jr, Amin MB, Egevad LL; ISUP Grading Committee. The 2005 International Society of Urological Pathology (ISUP) consensus conference on Gleason Grading of prostatic carcinoma. Am J Surg Pathol 2005;29:1228-42.

24. DeLong ER, DeLong DM, Clarke-Pearson DL. Comparing the areas under two or more correlated receiver operating characteristic curves: a nonparametric approach. Biometrics 1988;44:837-45.

25. Vickers AJ, Elkin EB. Decision curve analysis: a novel method for evaluating prediction models. Med Decis Making
2006;26:565-74.

26. Chiu PK, Lai FM, Teoh JY, Lee WM, Yee CH, Chan ES, et al. Prostate Health Index and \%p2PSA predict aggressive prostate cancer pathology in Chinese patients undergoing radical prostatectomy. Ann Surg Oncol 2016;23:2707-14.

27. Fossati N, Lazzeri M, Haese A, McNicholas T, de la Taille A, Buffi NM, et al. Clinical performance of serum isoform [-2] proPSA (p2PSA), and its derivatives \%p2PSA and the Prostate Health Index, in men aged $<60$ years: results from a multicentric European study. BJU Int 2015;115:913-20.

28. Semjonow A, Köpke T, Eltze E, Pepping-Schefers B, Bürgel $\mathrm{H}$, Darte C. Pre-analytical in-vitro stability of [-2]proPSA in blood and serum. Clin Biochem 2010;43:926-8.

29. Pandey RM. Approaches to sample size calculation in comparative studies. Indian J Pediatr 1999;66:533-8. 